\title{
General relativistic magnetic perturbations and dynamo effects in extragalactic radiosources
}

\author{
L. C. Garcia de Andrade ${ }^{1}$ \\ ${ }^{1}$ Departamento de Física Teórica-IF \\ Universidade do Estado do Rio de Janeiro \\ Rua São Francisco Xavier, 524 \\ Cep 20550-003, Maracanã, Rio de Janeiro, RJ, Brasil
}

\begin{abstract}
By making use of the MHD self-induction equation in general relativity (GR), recently derived by Clarkson and Marklund (2005), it is shown that when Friedmann universe possesses a spatial section whose Riemannian curvature is negative, the magnetic energy bounds computed by Nuñez (2002) also bounds the growth rate of the magnetic field given by the strain matrix of dynamo flow. Since in GR-MHD dynamo equation, the Ricci tensor couples with the universe magnetic field, only through diffusion, and most ages are highly conductive the interest is more theoretical here, and only very specific plasma astrophysical problems can be address such as in laboratory plasmas. Magnetic fields and the negative curvature of some isotropic cosmologies, contribute to enhence the amplification of the magnetic field. Ricci curvature energy is shown to add to strain matrix of the flow, to enhance dynamo action in the universe. Magnetic fluctuations of the Clarkson-Marklund equations for a constant magnetic field seed in highly conductive flat universes, leads to a magnetic contrast of $\frac{\delta B}{B} \approx 2$, which is well within observational limits from extragalactic radiosources of $\frac{\delta B}{B} \approx 1$.7. In the magnetic helicity fluctuations the magnetic contrast shows that the dynamo effects can be driven by these fluctuations.
\end{abstract}

\section{Introduction}

Since the early work by Gamow and Teller (1939), where they discovered an interesting relation between the Riemannian spatial hypersurfaces of negative curvature and the expansion of the open universe, this led naturally one to argue if that there is a similar relationship between there is a similar relation between the curvature and the expansion. In this paper, one shows that even when curvature vanishes, there is a constraint between the expansion and magnetic helicity. When the curvature of the spatial section is negative, a relationship is obtained between expansion, and Ricci curvature. Actually is easy to shown from dynamo equation that the expansion possesses a lower bound by the Ricci curvature. More recently, dynamo effects in cosmology have been adressed recently by Kleides et al.(2007), by using a anisotropic cosmological model in ideal highly conductive plasmas. In the realm of isotropic cosmology Brandenburg et al.(1998) have considered that decay of magnetic field can be slowed when turbulence is switch on. In this paper the isotropic case is further investigated in the case of hydromagnetic dynamo by making use of a recently derived, GR-MHD dynamo equation of Marklund and Clarkson (2005). This case is examined by using the approach developed by Nuñez (1995) in Sobolev spaces. Another example is that of a magnetic field helicity in cosmlogy. the paper is organized as follows: In section II the magnetic helicity model is addressed while in section III, the magnetic energy inequalities and the contribution of the Ricci term integral are given. 
Section IV addresses the issue of magnetic perturbations on extra radiogalactic sources. Conclusions are presented in section $\mathrm{V}$.

\section{Maqnetic helicity in non-ideal plasma cosmology}

Three dimensional dynamos in Riemannian space can be given by the self-induction equation (Arnold et al., 1981).

$$
\frac{\partial B}{\partial t}=-\{v, B\}+\operatorname{div}(v) B+\eta \Delta B
$$

where $\eta$ is the plasma resistivity and $\Delta:=\nabla^{2}$ is the Laplacian operator, where $\{v, B\}=$ $-\operatorname{curl}(v \times B)$. In Chicone et al.(1999) the Riemannian manifold was confined to incompressible dynamo flows, where the second term on the RHS of expression (3.2) vanishes, and along with the divergence-free condition of the magnetic field

$$
\operatorname{div} B=0
$$

they form a solenoidal vector field in Riemannian manifold. On the other MHD-GR dynamo equation in pseudo-Riemannian spacetime given by its 3-spatial section

$$
\dot{\mathbf{B}}-\nabla \times[\mathbf{v} \times \mathbf{B}]-\eta \Delta \mathbf{B}=-\eta\left[\frac{2}{3} \theta \mathbf{B}-\text { Ric. } \mathbf{B}\right]-\left(1+\frac{2}{3} \eta \theta\right) \frac{2}{3} \theta \mathbf{B}-\eta \nabla \times(\mathbf{a} \times \mathbf{B})+\eta \Theta
$$

where Ric represents Ricci tensor and $\theta$ is the expansion of the Friedmann universe. In this case shear and global vorticity vanish. As is easily noted from this equation, the Ricci curvature has no effect in dynamo action in most highly conductive cosmological models, since then the diffusion constant cefficient $\eta$ vanishes. The symbol $\Theta$ is given by

$$
\Theta=-\frac{2}{3} \dot{\theta} \mathbf{B}-\frac{2}{3} \dot{\theta} \dot{\mathbf{B}}-\mathbf{a} \times\left[\nabla \times \mathbf{B}-\mu_{0} \mathbf{j}+\mathbf{a} \times \mathbf{B}\right]
$$

By Fourier analyzing the above equation by the expression

$$
\mathbf{B}=\mathbf{B}_{0} \exp [\gamma t-i \mathrm{kx}]
$$

yields

$$
\operatorname{div} \mathbf{B}=i \mathbf{k} \cdot \mathbf{B}=0
$$

for the solenoidal equation and

$$
\begin{aligned}
& \mathbf{k} \times(\mathbf{v} \times \mathbf{B})=0 \\
& \mathbf{a} \times(\mathbf{a} \times \mathbf{B}) \approx 0
\end{aligned}
$$

where in the last equation one had assumed that the acceleration of the universe $\mathbf{a}$ is low. Magnetic helicity is given by the constraint

$$
\nabla \times \mathbf{B}=\lambda \mathbf{B}
$$

and it is used in the above computations yields

$$
\dot{\mathbf{B}}-\nabla \times[\mathbf{v} \times \mathbf{B}]+\eta \lambda^{2} \mathbf{B}=-\eta\left[\frac{2}{3} \theta \mathbf{B}-\text { Ric. } \mathbf{B}\right]-\left(1+\frac{2}{3} \eta \theta\right) \frac{2}{3} \theta \mathbf{B}-\eta \nabla \times(\mathbf{a} \times \mathbf{B})+\eta \Theta
$$

In the flat Friedmann universe, where the spatial section of the spacetime is zero, $\mathrm{r}$ Ric $=0$, this equation reduces to the one

$$
\frac{1}{2} \dot{\mathbf{B}^{2}}=\eta\left[\lambda^{2}+\frac{4}{9} \theta^{2}-\frac{2}{3} \dot{\theta}\right] \mathbf{B}^{2}-\frac{2}{3} \theta \mathbf{B}^{2}
$$


where the convective term $\nabla \times[\mathbf{v} \times \mathbf{B}$ vanishes since by Fourier transformation

$$
\mathbf{k} \times[\mathbf{v} \times \mathbf{B}]=i[(\mathbf{k} . \mathbf{B}) \mathbf{v}-(\mathbf{k . v}) \mathbf{B}]
$$

since both velocity and magnetic fields are solenoidal. Then, considering that the magnetic field decays as $B \approx s^{-2}$, one obtains the self-induction equation as

$$
\dot{\theta_{1}}-\frac{9}{4} \theta_{1}=0
$$

where the old expansion is related to the new as

$$
\theta=\theta_{1}-\frac{2}{3} \lambda^{2}
$$

Therefore solution of equation (2.13) is

$$
\theta=\theta_{0} \sinh \left[\frac{3}{2} t\right]+\frac{2}{3} \lambda^{2}
$$

which shows that magnetic helicity enhances the expansion of the universe, when magnetic field decay. Now let us briefly examine and put some bounds on the growth of the magnetic field, when the Riemannian spatial curvature of the spatial section is negative as in 3D case of fast dynamos in Riemannian compact manifolds by Chicone and Latushkin (1999). In this case the FRW metric is open and the self-induction equation is

$$
\frac{\dot{B}}{B}=\eta\left[-\lambda^{2}+\frac{2}{3} \dot{\theta}-R i c-\frac{4}{9} \theta^{2}\right]-\frac{2}{3} \theta \geqslant 0
$$

the equal sign corresponds to marginal dynamos. Since Ric $\leqslant 0$, in the case of Riemannian negative curvature,the Ric term contributes to enhance the dynamo effect as in Kleidis et al result, but basic difference is that here the plasma is non-ideal and the spacetime isotropic since one imagines, that here dynamo is kinematic and not hydromagnetic as in the next section. In the case of negative Riemann spatial or Gaussian Ricci curvature, and the constraint of slow expansion, $\theta<<1$ and $\dot{\theta}<<1$ yields

$$
\text { Ric }<\frac{3}{2} \eta \theta
$$

which yields an explicitly constraint between expansion the diffusion and the Ricci scalar.

\section{Bounds on hydromagnetic dynamo growth rate cosmology}

In this section one shall consider the bounds on the magnetic energy of the hydromagnetic dynamos in spatially curved cosmlogical isotropic models. One shall demonstrate from the above GR-MHD relativistic plasma dynamo equations for finite magnetic Reynolds numbers $R_{m}=\eta^{-1}$, the Ricci scalar volume integral is fundamental for these bounds. Let us consider the magnetic energy time evolution as

$$
\frac{1}{2} \frac{\partial \int B^{2} d V}{\partial t}=\int(\mathbf{B} \cdot \nabla) \mathbf{v} \cdot \mathbf{B} d V-\eta \int|\nabla B|^{2} d V-\eta \int R i c \cdot \mathbf{B} \cdot \mathbf{B} d V
$$

Since under the plasma resistivity, the only new term compared to non-GR plasmas is the one with Ricci tensor, then to investigate the magnetic energy bounds, it is enough to investigate this bound contribution as

$$
-\eta \int R i c . \mathbf{B} \cdot \mathbf{B} d V=-\eta \int R_{i j} B^{i} B^{j} d V=-6 \eta \int \frac{K}{a^{2}} \delta_{i j} B^{i} B^{j} d V=+6 \eta \int \frac{1}{a^{2}} B^{2} d V \geqslant 0
$$


where $\mathrm{K}$ is the Gaussian negative curvature of the negatively curved Riemannian spatial section like in Gamow-Teller universe. Thus since this contribution is positive, when the universe shrinks or collapses the curvature term will greatly enhance dynamo action and the bounds for the growth rate. Since, as shown by Nuñez (2002) in his study of the dissipation of kinetic energy in the hydromagnetic dynamo, the following inequality applies

$$
\left.\left|\int \mathbf{B} \cdot \nabla \mathbf{v} \cdot \mathbf{B} d V\right| \leqslant \frac{1}{2}\left\|(\nabla \mathbf{v})^{t}+\nabla \mathbf{v}\right\|_{\infty}\right) \int B^{2} d V
$$

Inserting the new Ricci curvature energy into this expression yields

$$
\gamma \leqslant\left(\frac{1}{2}\left\|(\nabla \mathbf{v})^{t}+\nabla \mathbf{v}\right\|_{\infty}+<\frac{6 \eta}{a^{2}}>\right)
$$

where to obtain this term, one had to assume that the gradients of the magnetic field would be highly suppressed by the overall magnetic energy at all. The brackets on the RHS of the inequality means a mean of averaged value over the volume of a small portion of the universe. Thus in general the Ricci curvature adds to strain matrix to enhance dynamo action in the presence of diffusion. This seems to agree with Kleides et al result in the case of diffusive isotropic cosmolgical models.

\section{Magnetic fluctuations and dynamo effects in GR extragalactic radiosources}

As pointed out by Ruzmaikin et al.(1981), there is a gap between the observational value of the contrast obtained by the joint catalogue of polarization properties of extra galactic radiosources $\frac{\delta B}{B} \approx 1.7$ and the one estimated by Ruzmaikin et al.(1981) as $\frac{\delta B}{B} \approx 10^{3}$. In this section an estimate more realistic and well within observational values is given by the contrast $\frac{\delta B}{B} \approx 2$. These computations are performed in the magnetic helicity-free case. To start let us use the magnetic field perturbation given by

$$
B=B_{0}+\delta B
$$

where $\delta B$ is the magnetic fluctuation and $B_{0}$ is here the constant magnetic field that may seed the galactic dynamo. Substitution of this fluctuation into the above expression for the magnetic energy density, in the case of a highly conducting $(\eta=0)$ universe yields

$$
\frac{\delta B}{B}=1+\exp \left[-\frac{2}{3} \int \delta \theta d t\right]
$$

where one has addopted a constant initial expansion of the universe, to simplify computations. For a very small universe fluctuation in expansion and for a finite time interval, this result reduces to

$$
\left.\frac{\delta B}{B}=2-\frac{2}{3} \int \delta \theta d t\right] \approx 2
$$

as one wishes to prove. Let us now consider the case where the magnetic helicity fluctuations are present. In this case computations leads to

$$
\frac{\delta B}{B}=\frac{1}{\sqrt{2+\frac{1}{3} \delta \theta \eta}} \delta \lambda
$$

which reduces to

$$
\frac{\delta B}{B}=\frac{1}{\sqrt{2 \eta}} \delta \lambda
$$


This last expression can be used to place a limit on magnetic helicity from the magnetic contrast and magnetic Reynolds numbers.

\section{Conclusions}

Gravitational magnetic fluctuations in terms of universe expansin fluctuations can be obtained from GR-MHD dynamo equation. Physical applications lead us to find contrasts to the magnetic fields or magnetic field fluctuations that are well within experimental values for the extra galactic radio sources. Bounds for hydromagnetic energy based on these gr dynamo equation are also found.

\section{Acknowledgements}

Several discussions with A Brandenburg, Yu Latushkin, D Sokoloff and R Beck are highly appreciated. I also thank financial supports from UERJ and CNPq.

\section{References}

Gamow, G. \& Teller, E., 1939, Phys. Rev.; Widrow, L. Rev. Mod. Phys., 2002, 74, 75.

Kleides, K., Kuiroikidis, A., Papadopoulos D., \& Vlahos, F., arxiv 07124239.

Brandenburg, A., 1998, Phys. Rev. D

Marklund, M. \& Clarkson, C. 2005, MNRAS, 358, 892.

Nuñez, M., J Math Phys. 2003, 38,1538 (1997). Nuñez, M., J Phys A,8903.

Arnold, V., Zeldovich, Y. B., Ruzmaikin, A., \& Sokoloff, D. D., 1981, JETP, 1981, 81 \& 1982, Doklady Akad. Nauka, SSSR, 266, 1357.

Chicone C. \& Latushkin, Y., 1999, American Mathematical Society, AMS.

Nuñez, M. 2002, Geophys and Astr. Fluid Dynamics, 96, 345. 